\title{
Improving patient safety in critical care: big chal- lenge, exciting opportunity
}

Peter Dodek MD MHSC

A

WARENESS of patient safety as an important quality of care problem has been heightened by the recent publication of several studies of medical error and adverse events in Canadian hospitals. ${ }^{1-5}$ In the largest and most recent of these studies, Baker and colleagues found an average of 7.5 adverse events per 100 hospital admissions in 20 hospitals from across Canada; ${ }^{1}$ $36.9 \%$ of those adverse events were preventable. Given the increased severity of illness and frequency of pharmacologic and other interventions in intensive care units (ICUs), critically ill patients are especially prone to medical errors and adverse events. ${ }^{6-8}$ These adverse events include not only adverse medication events but also complications of technological interventions such as ventilator-associated pneumonia (VAP). ${ }^{9}$

VAP occurs in 10 to $65 \%$ of ventilated patients and has an attributable morbidity of 4.3 additional ICU days. In this issue of the Journal, Baxter and colleagues report their results of introducing a multifaceted protocol to prevent VAP in a single ICU. ${ }^{10}$ They found that the incidence of VAP in their ICU decreased from 26.7 cases $/ 1,000$ ventilator days to 12.5 cases/1,000 ventilator days, an impressive change. Although there were no other known causes of this change, it would have been useful to see measures of the risk factors for VAP (especially considering the change in case mix during the course of this study) and process of care (did the health care professionals actually do what they said they would do?). Strengths of this study include the use of evidence-based guidelines as a basis for the planned changes, ${ }^{11,12}$ and a multidisciplinary and multi-pronged approach to development and implementation of these changes. ${ }^{13}$

Reducing the incidence of VAP is both a big challenge and an exciting opportunity. Given the current state of evidence, there are several relatively easy strategies to accomplish this goal. For example, there is little effort and no additional cost required in using the orotracheal instead of the nasotracheal route for intubation, eliminating scheduled changes of ventilator circuits, placing patients in a semi-recumbent position, ${ }^{12}$ or using enteral instead of parenteral feeding. ${ }^{11}$ For other strategies that involve new equipment such as subglottic secretion drainage, the incremental cost of the new equipment (a few dollars per modified endotracheal tube in this case) is dwarfed by the savings accrued by preventing a case of VAP. The number of modified endotracheal tubes needed to prevent one episode of VAP is about eight. ${ }^{14}$

Other challenges in improving safety in critical care include decreasing the number of avoidable transfusions of packed red blood cells ${ }^{15}$ and minimizing harm due to high-risk medications such as insulin, heparin, sedatives, and analgesics. In both of these examples, the exciting opportunity is that we need to do less, not more. This does not require extra money, but it does require will and commitment. Unfortunately, will and commitment to patient safety are easily diluted by other distractions. One approach to galvanizing collective will and to maintaining constancy of purpose is to collaborate in improvement efforts with other institutions. ${ }^{16}$ This is exactly what is currently happening among more than 30 ICU teams from across Canada who are part of the Canadian Collaborative to Improve Patient Care and Safety in ICUs. These teams are using an explicit approach to incremental improvement ${ }^{17}$ to decrease the incidence of VAP, decrease avoidable transfusions of packed red cells, minimize harm from high-risk medications, decrease mortality from severe sepsis, and decrease cardiac arrests through the use of medical emergency teams. This collaborative has been operational since June 2003 and several teams have already made remarkable improvements.

From the Centre for Health Evaluation and Outcome Sciences and Program in Critical Care Medicine, St. Paul's Hospital, University of British Columbia, Vancouver, British Columbia, Canada.

Address correspondence to: Dr. Peter Dodek, Center for Health Evaluation and Outcome Sciences, St. Paul's Hospital, 1081 Burrard

Street, Vancouver, British Columbia V6Z 1Y6, Canada. Phone: 604-806-9023; Fax: 604-806-8674; E-mail: pedodek@interchange.ubc.ca 
A collaborative approach to improvement is part of a culture change that is also necessary to ensure safety. Although there is not yet published evidence that organizational culture is directly linked to safety in health care, ${ }^{18}$ evidence from other industries including the airline industry ${ }^{19}$ indicates that there is a direct relationship between employee perception of organizational culture and safety events. Once again, the challenge is big but the opportunity is exciting because there are several simple strategies to develop a 'culture of safety' in which "all staff members can talk freely about safety problems and how to solve them, without fear of blame or punishment." ${ }^{20}$ These include initiating safety briefings in which small groups of staff members meet regularly for five minutes to informally address two questions: are you aware of any adverse events or near misses? Do you have any suggestions to improve patient safety? Other simple strategies include Patient Safety Leadership WalkRounds ${ }^{\mathrm{TM}}$ in which health care leaders meet briefly and informally with front-line health care workers to ask questions about patient safety in the workplace. Other important strategies to develop a 'culture of safety' include the development of a safety reporting system ${ }^{21}$ and the designation of a patient safety officer.

As Baxter and colleagues have illustrated in their study, ${ }^{10}$ improvement of just one patient safety problem, VAP, is a big challenge. However, their study also illustrates the exciting opportunity to use evidencebased recommendations for practice and implementation, and simple educational and leadership strategies to build a 'culture of safety.'

\section{L'amélioration de la sécurité des patients à l'unité des soins inten- sifs : un grand défi, une occasion stimulante}

La reconnaissance de la sécurité du patient comme qualité importante des soins a été intensifiée par des études récentes sur des erreurs médicales et des événements indésirables survenus dans les hôpitaux canadiens. ${ }^{1-5}$ Dans la plus importante et la plus récente étude, Baker et coll. ont trouvé une moyenne de 7,5 événements indésirables par 100 admissions dans 20 hôpitaux canadiens $;^{1} 36,9 \%$ de ces incidents étaient évitables. Comme la sévérité des maladies et de la fréquence des interventions pharmacologiques, et autres, augmentent dans les unités de soins intensifs (USI), les grands malades sont particulièrement exposés aux erreurs médicales et aux événements indésirables. ${ }^{6-8}$ Ces derniers comptent non seulement des erreurs de médication, mais aussi des complications d'interventions technologiques comme la pneumonie associée à la ventilation mécanique (PAVM). ${ }^{9}$

La PAVM survient chez 10 à $65 \%$ des patients ventilés et présente une morbidité attribuable de 4,3 jours additionnels à l'USI. Dans le présent numéro du Journal, Baxter et ses collègues présentent leurs résultats sur l'introduction d'un protocole à plusieurs facettes visant à prévenir la PAVM dans une seule USI. ${ }^{10}$ Ils ont trouvé que l'incidence de PAVM dans leur USI avait baissé de 26,7 cas/1 000 jours de ventilation à 12,5 cas $/ 1000$ jours de ventilation, une amélioration impressionnante. Bien que ce changement ne semble pas relever d'autres causes connues, il aurait été intéressant de voir la mesure des facteurs de risque de PAVM, en tenant compte surtout de la variation des cas pendant l'étude, et des modalités de traitement - les professionnels de la santé font-ils vraiment ce qu'ils disent faire ? Les forces de cette étude sont l'usage de directives fondées sur des preuves comme base des changements planifiés ${ }^{11,12}$ et une approche multidisciplinaire et concertée pour le développement et l'application de ces changements. ${ }^{13}$

La réduction de l'incidence de PAVM est un grand défi et une occasion stimulante. Certaines stratégies relativement faciles permettent d'atteindre ce but. Par exemple, il n'y a qu'un petit effort à faire et aucun coût additionnel n'est nécessaire pour procéder à une intubation orotrachéale plutôt que nasotrachéale, éliminer les changements programmés de circuits de ventilation, placer le patient en position semi-assise ${ }^{12}$ ou choisir la nutrition entérale plutôt que parentérale. ${ }^{11}$ Pour d'autres stratégies avec du nouveau matériel, comme le drainage des sécrétions sous-glottiques, le coût incrémentiel du matériel (quelques dollars par tube endotrachéal modifié dans ce cas) est amoindri par les épargnes acquises de la prévention d'un cas de PAVM. Le nombre de tubes endotrachéaux modifiés, nécessaires pour prévenir un épisode de PAVM, est d'environ huit. ${ }^{14}$

L'amélioration de la sécurité aux soins intensifs porte aussi sur la baisse du nombre de transfusions évitables de culots globulaires ${ }^{15}$ et la réduction d'effets nuisibles relatifs aux médicaments à haut risque comme l'insuline, l'héparine, les sédatifs et les anal- 
gésiques. Dans les deux cas, il est stimulant que nous n'ayons qu'à en faire moins, non plus. Cela n'exige pas plus d'argent, mais nécessite volonté et engagement. Malheureusement, la volonté et l'engagement d'agir pour la sécurité du patient sont facilement dilués par d'autres distractions. Une approche qui pourrait animer la volonté collective et maintenir la constance des objectifs serait de collaborer avec d'autres institutions. ${ }^{16}$ C'est ce qui arrive présentement dans plus de 30 équipes d'USI à travers le Canada qui font partie de la Collaboration canadienne pour l'amélioration des soins et de la sécurité des patients dans les USI. Ces équipes utilisent une approche explicite d'amélioration graduelle ${ }^{17}$ pour réduire l'incidence de PAVM, les transfusions évitables de culots globulaires, les effets négatifs de médicaments à haut risque, la mortalité causée par une sévère septicémie et le nombre d'arrêts cardiaques par l'intervention d'équipes d'urgence médicale. Cette collaboration existe depuis juin 2003 et quelques équipes ont déjà fait de remarquables progrès.

La collaboration à l'amélioration fait partie d'un changement culturel nécessaire aussi pour assurer la sécurité. Même s'il n'y a pas encore de preuve publiée que la culture organisationnelle soit directement liée à la sécurité des soins de santé, ${ }^{18}$ d'autres industries, incluant l'aviation commerciale, ${ }^{19}$ ont montré qu'il y a une relation directe entre la perception que les employés ont de la culture organisationnelle et la situation de sécurité. Une fois encore, il y a des stratégies simples pour développer une "culture de sécurité» dans laquelle «tous les membres du personnel peuvent parler librement des problèmes de sécurité et de la façon de les résoudre, sans crainte de blâme ou de sanction». ${ }^{20}$ Cela comprend l'introduction de brefs sur la sécurité où de petits groupes de membres du personnel se rencontrent régulièrement pendant cinq minutes pour répondre à deux questions de façon informelle : êtes-vous au courant d'événements indésirables ou presque catastrophiques ? Avez-vous des suggestions pour améliorer la sécurité des patients ? D'autres stratégies simples visent les Patient Safety Leadership WalkRounds ${ }^{\mathrm{TM}}$ où les responsables des soins de santé rencontrent brièvement et de manière informelle le personnel de la santé de première ligne et s'informent sur la sécurité des patients dans le milieu de travail. D'autres stratégies importantes incluent le développement d'un système de déclaration d'incident ${ }^{21}$ et la désignation d'un responsable de la sécurité des patients.

Comme Baxter et ses collègues l'ont illustré, ${ }^{10}$ l'amélioration d'un seul problème de sécurité, la PAVM, est un grand défi. Cependant, leur étude mon- tre aussi une occasion stimulante d'utiliser des recommandations d'exercice fondées sur des preuves et des stratégies simples de formation et de direction pour construire une «culture de sécurité».

\section{References}

1 Baker GR, Norton PG, Flintoft V, et al. The Canadian Adverse Events Study: the incidence of adverse events among hospital patients in Canada. CMAJ 2004; 170 : 1678-86.

2 Choi PP, Day A, Etchells E. Gaps in the care of patients admitted to hospital with an exacerbation of chronic obstructive pulmonary disease. CMAJ 2004; 170: 1409-13.

3 Forster AJ, Asmis TR, Clark HD, et al. Ottawa Hospital Patient Safety Study: incidence and timing of adverse events in patients admitted to a Canadian teaching hospital. CMAJ 2004; 170: 1235-40.

4 Proctor ML, Pastore J, Gerstle JT, Langer JC. Incidence of medical error and adverse outcomes on a pediatric general surgery service. J Pediatric Surg 2003; 38 : 1361-5.

5 Orser BA, Chen RJ, Yee DA. Medication errors in anesthetic practice: a survey of 687 practitioners. Can J Anesth 2001; 48: 139-46.

6 Donchin $\Upsilon$, Gopher D, Olin M, et al. A look into the nature and causes of human errors in the intensive care unit. Crit Care Med 1995; 23: 294-300.

7 Andrews LB, Stocking C, Krizek T, et al. An alternative strategy for studying adverse events in medical care. Lancet 1997; 349: 309-13.

8 Osmon S, Harris CB, Dunagan WC, Prentice D, Fraser VJ, Kollef $M H$. Reporting of medical errors: an intensive care experience. Crit Care Med 2004; 32: 727-33.

9 Keenan SP, Heyland DK, Jacka MJ, Cook D, Dodek P. Ventilator-associated pneumonia. Prevention, diagnosis, and therapy. Crit Care Clin 2002; 18: 107-25.

10 Baxter AD, Allan J, Bedard J, et al. Adherence to simple and effective measures reduces the incidence of ventilator-associated pneumonia. Can J Anesth 2005; 52: 535-41.

11 Heyland DK, Dhaliwal R, Drover JW, Gramlich L, Dodek P; Canadian Critical Care Clinical Practice Guidelines Committee. Canadian clinical practice guidelines for nutrition support in mechanically ventilated, critically ill adult patients. JPEN J Parenter Enteral Nutr 2003; 27: 355-73.

12 Dodek P, Keenan S, Cook DJ, et al; Canadian Critical Care Trials Group and the Canadian Critical Care Society. Evidence-based clinical practice guideline for the prevention of ventilator-associated pneumonia. Ann Intern Med 2004; 141: 305-13.

13 Grimshaw JM, Shirran L, Thomas R, et al. Changing 
provider behavior: an overview of systematic reviews of interventions. Med Care 2001; 39(Suppl 2): II-2-II-45.

14 Smulders $K$, van der Hoeven H, Weers-Pothoff I, Vandenbrouke-Grauls C. A randomized clinical trial of intermittent subglottic secretion drainage in patients receiving mechanical ventilation. Chest $2002 ; 121$ : 858-62.

15 Corwin HL, Gettinger A, Pearl RG, et al. The CRIT Study: anemia and blood transfusion in the critically ill-current clinical practice in the United States. Crit Care Med 2004; 32: 39-52.

16 Weeks WB, Mills PD, Dittus RS, Aron DC, Batalden $P B$. Using an improvement model to reduce adverse drug events in VA facilities. Joint Comm J Qual Imp 2001; 27: 243-54.

17 Langley GJ, Nolan KM, Nolan TW, Norman CL, Provost LP. The Improvement Guide: A Practical Approach to Enhancing Organizational Performance. San Francisco, CA: Jossey-Bass; 1996.

18 Hoff T, Jameson L, Hannan E, Flink E. A review of the literature examining linkages between organizational factors, medical errors, and patient safety. Medical Care Res Rev 2004; 61: 3-37.

19 Sexton JB, Klinect JR. The link between safety attitudes and observed performance in flight operations.

Proceedings of the 1lth International Symposium on Aviation Psychology, 2001. Columbus, OH, The Ohio State University, 2001.

20 Institute for Heathcare Improvement. Develop a culture of Safety. http://www.ihi.org/IHI/Topics/ PatientSafety/SafetyGeneral/Changes/Develop+a+Cul ture+of+Safety.htm; accessed on September 15, 2004.

21 Lubomski LH, Pronovost PJ, Thompson DA, et al. Lessons from the Intensive Care Unit Safety Reporting System (ICUSRS). J Clin Outcomes Management 2004; 11: 257-80. 